\title{
CYCLOTRON AUTORESONANCE ACCELERATOR FOR ELECTRON BEAM DRY SCRUBBING OF FLUE GASES ${ }^{*}$
}

\author{
J. L. Hirshfield ${ }^{1,2}$ and Changbiao Wang ${ }^{1}$ \\ ${ }^{1}$ Physics Department, Yale University, New Haven, Connecticut 06520-1820 \\ ${ }^{2}$ Omega-P, Inc., 202008 Yale Station, New Haven, Connecticut 06520
}

\begin{abstract}
A self-scanning, highly-efficient electron beam source is being built for electron beam dry scrubbing (EBDS) of flue gases. The beam is to be prepared using cyclotron autoresonance acceleration (CARA), which has already experimentally demonstrated an rf efficiency above $90 \%$. The design is based on simulation studies for a $250-\mathrm{kV}$, 25 -A warm injected beam which is accelerated in a 112$\mathrm{cm}$ long CARA using $21 \mathrm{MW}$ of rf power at $2.856 \mathrm{GHz}$. The accelerated gyrating beam has an energy of nearly 1.1 $\mathrm{MeV}$, with a theoretical acceleration efficiency of $99 \%$. The beam can be extracted from CARA through a foil window, once the axial magnetic field is down-tapered to zero, and self-scan on a conical surface across a rising flue gas stream in a field-free region.
\end{abstract}

\section{INTRODUCTION}

Existing environmental anti-pollution regulations provide strong impetus for development of cost-effective technology to reduce acid rain through removal of sulfur and nitrogen oxides from flue gas releases to the atmosphere [1]. Limestone slurry scrubbing and selective catalytic reduction are the conventional technologies for flue gas cleanup [2]. An alternative technology, electron beam dry scrubbing (EBDS), was introduced in 1970, and subsequently demonstrated in several manifestations [3]. Perhaps lacking in encouraging EBDS deployment is the existence of a compact, low-cost electron beam source of high average power and high wall plug efficiency.

Cyclotron autoresonance acceleration (CARA) is an efficient process for converting rf energy into electron beam energy [4,5]. Based on existing $\mathrm{rf}$ source and electron gun technology, CARA can provide a high average power gyrating electron beam. Recent CARA experiments have shown that over $90 \%$ of the input if power can be transferred to an electron beam [6]. The accelerated beam produced in CARA emerges on a gyrating trajectory; thus the beam is "self-scanning", requiring no other deflecting device or external field to sweep across a gas stream. It is this unique feature that makes CARA particularly suitable for EBDS, and perhaps other radiation chemistry applications.

An upgrade of the Yale/Omega-P CARA is being built that is designed to produce a $25 \mathrm{~A}, 0.8-1.2 \mathrm{MeV}$ electron beam, using up to $20 \mathrm{MW}$ of $2.856 \mathrm{GHz}$ rf power and injection up to $300 \mathrm{kV}$. Here, analysis is presented underlying this design, using $250 \mathrm{kV}$ injection.

\section{BASIC PRINCIPLES}

A comprehensive theoretical analysis of CARA with supporting particle simulation studies [5] has been carried out based on prior work [4]. The basic idea of such an accelerator is that a pencil electron beam from a convergent-flow Pierce gun is passed through a cylindrical waveguide. $T E_{11}$ rotating mode $\mathrm{rf}$ drive power energizes the beam by cyclotron autoresonance. The synchronous axial magnetic field for the interaction is given by

$$
B_{o}=\frac{m_{o}}{e} \omega \gamma\left(1-n \beta_{z}\right),
$$

where $\omega$ is the drive frequency, $e$ and $m_{o}$ are the electron's charge and rest mass, $\gamma$ is the relativistic energy factor, $\beta_{z}$ is the axial velocity normalized to the light speed $c$ in free space, and the index of refraction (normalized group velocity) for the $T E_{11}$ mode is $n=k_{z} c / \omega$, with $k_{z}$ the axial wave number. As $\nabla \cdot \mathbf{B}=0$ requires, the transverse magnetic field increases when the axial magnetic field increases, as the electron's acceleration energy increase: this reduces the electron's axial velocity towards stalling, as in the mirror effect. This imposes a maximum energy that can be reached in CARA, given by

$$
\gamma_{\max }=\gamma_{o}+\left(\frac{\gamma_{o}^{2}-1}{1-n^{2}}\right)^{1 / 2}
$$

where $\gamma_{o}$ is the energy factor of the injected beam. The electron's gyration radius is constrained by the synchronous condition Eq. 1, which results in a maximum gyration radius, given by

$$
\frac{r_{L \max }}{R_{w}}=\frac{1}{j_{11}^{\prime}}\left[\frac{\left(\gamma_{\max }^{2}-1\right)\left(1-n^{2}\right)}{\left(\gamma_{\max }^{2}-1\right)\left(1-n^{2}\right)+1}\right]^{1 / 2}<0.5431,
$$

where $r_{L \max }$ is the maximum gyration radius, $R_{w}$ is the waveguide radius, and $j_{11}^{\prime}=1.841184$ is the first root of the Bessel function derivative $J_{1}^{\prime}(\chi)$. Eq. 3 states that the normalized gyration radius must be less than 0.5431 regardless of the beam's energy or the waveguide radius.

Solving Eq. 2 for $\gamma_{o}$, we obtain

$$
\gamma_{o}=\frac{1}{n^{2}}\left\{\left[\gamma_{\max }^{2}\left(1-n^{2}\right)+n^{2}\right]^{1 / 2}-\gamma_{\max }\left(1-n^{2}\right)\right\} \text {. }
$$

Eq. 4 is helpful in obtaining CARA design parameters. For a $1.0 \mathrm{MV}, 25 \mathrm{~A}$ output electron beam,

\footnotetext{
* The work was supported by the U. S. Department of Energy, Divisions of High Energy Physics and Advanced Energy Projects.
} 
for example, when one sets $\gamma_{\max }=2.9569$, one finds $\gamma_{o}=1.3896$, or an initial beam voltage of $199 \mathrm{kV}$. This is for $n=0.7880$ ( $R_{w}=5.0 \mathrm{~cm}$ if the drive frequency is $2.856 \mathrm{GHz}$ ). An input drive power of $20 \mathrm{MW}$ is required. In practice, the initial beam voltage should be larger than $199 \mathrm{kV}(250 \mathrm{kV}$, for example), to not approach stalling.

\section{SIMULATION RESULTS}

Here we present simulation results for a CARA to show that existing rf source and electron gun technology can allow acceleration of a beam to about $1 \mathrm{MV}$, as needed for EBDS. In the simulation, a single-energy injected electron beam is assumed, with a guiding center spread of $10 \%$ and an rms axial velocity spread of $0.02 \%$. This velocity spread value is scaled from that for the $100 \mathrm{kV}$ gun now in operation on the Yale/Omega-P CARA. The number of computational particles is 128 , with 8 values of velocity spread, 8 values of phase spread, and 2 values of guiding center spread. Simulation parameters are given in Table I.

TABLE I. Parameters in simulation.

\begin{tabular}{lc}
\hline injection gun voltage & \multicolumn{2}{c}{$\begin{array}{l}250 \mathrm{kV} \\
\text { injector gun perveance }\end{array}$} & $\begin{array}{l}0.2 \times 10^{-6} \mathrm{~A}-\mathrm{V}^{-3 / 2} \\
\text { beam current }\end{array}$ & $25 \mathrm{~A}$ \\
rms axial velocity spread & $0.02 \%$ \\
beam guiding center spread $10 \%$ & \\
rf drive frequency & $2.856 \mathrm{GHz}$ \\
rf drive power & $21 \mathrm{MW}$ \\
guide magnetic field & $<1.8 \mathrm{kG}$ \\
\hline
\end{tabular}

The waveguide of the CARA operating in the rotating $T E_{11}$ mode consists of three sections. The first is an input rf coupler which has a radius of $3.3 \mathrm{~cm}$ and a length of $12 \mathrm{~cm}$, the third has a radius of $5 \mathrm{~cm}$ and a length of 60 $\mathrm{cm}$, and they are uniform. The second is a $40-\mathrm{cm}$ long taper. The taper's slope is only 0.0425 (about 2.4 degrees), and wave reflection is ignored in the simulation. Following CARA, there is a $60-\mathrm{cm}$ long drift region, where there is no rf field and the axial magnetic field is linearly tapered down to zero.

Fig. 1 shows the dependence of normalized average gyration radius $\left\langle r_{L} / R_{w}\right\rangle$ on axial distance $z$ for the parameters in Table I, for comparison with the normalized maximum gyration radius $r_{L \max } / R_{w}$ calculated from Eq. 3 , and maximum acceleration energy $\gamma_{\max }$ from Eq. 2. For the first and third sections, the waveguide radius is uniform and $\gamma_{\max }$ stays constant, with $\gamma_{\max }=2.6723$ in the former and $\gamma_{\max }=3.2817$ in the latter; for the second section, $\gamma_{\max }$ increases as the radius increases. Clearly, it can be seen that the computed gyration radius $<r_{L} / R_{w}>$ is less than $r_{L \max } / R_{w}(<0.5431)$.

The rf input coupler is intended for use in common with a $20-\mathrm{GHz} 7$ th harmonic co-generation experiment that requires a $3.3-\mathrm{cm}$ radius waveguide [7]. From Fig. 1 we see that the maximum acceleration energy for $3.3 \mathrm{~cm}$ is only $855 \mathrm{kV}\left(\gamma_{\max }=2.6723\right)$ for the $250 \mathrm{kV}$ injected beam, which is marginally low to be suitable for an EBDS CARA. The waveguide taper is designed to increase the maximum acceleration energy by increasing the radius from $3.3 \mathrm{~cm}$ to $5.0 \mathrm{~cm}$. This allows the maximum acceleration energy at the end of CARA to reach $1166 \mathrm{kV}\left(\gamma_{\max }=3.2817\right)$.

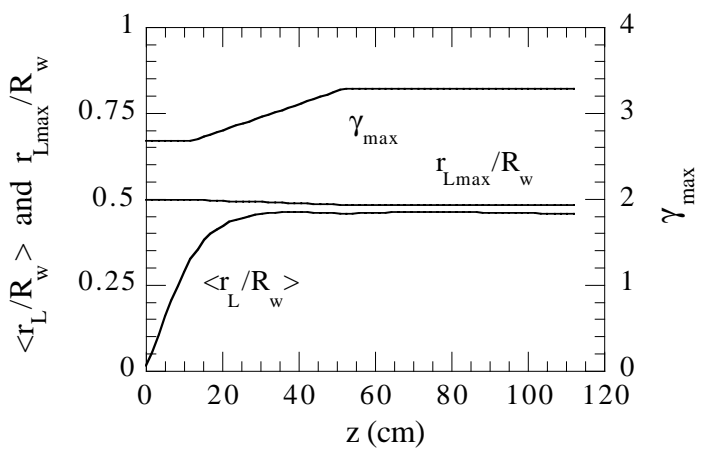

Figure 1: Average gyration radius $\left\langle r_{L} / R_{w}>\right.$, maximum gyration radius $r_{L \max } / R_{w}$, and maximum acceleration energy $\gamma_{\max }$ versus axial distance $z$.

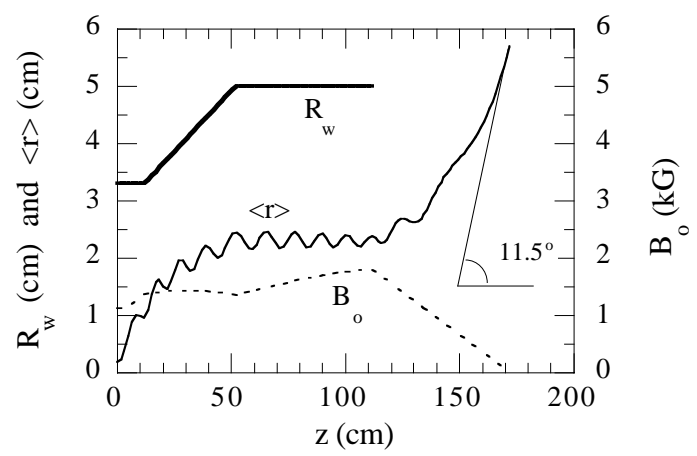

Figure 2: Waveguide radius $R_{w}$, electron's average radial coordinate $\langle r\rangle$, and axial magnetic field $B_{o}$ versus $z$.

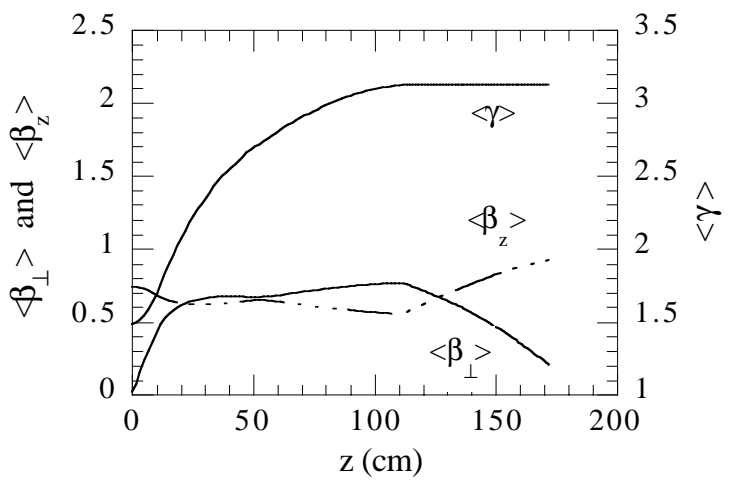

Figure 3: Dependence of normalized average transverse velocity $\left\langle\beta_{\perp}>\right.$ and axial velocity $\left\langle\beta_{z}>\right.$, and relativistic energy factor $\langle\gamma>$ on axial distance $z$.

Fig. 2 shows the dependence of waveguide radius $R_{w}$, electron's average radial coordinate $\langle r\rangle$, and axial magnetic field $B_{o}$ on axial distance $z$, while Fig. 3 shows the dependence of normalized average transverse 
velocity $\left\langle\beta_{\perp}>\right.$ and axial velocity $\left\langle\beta_{z}\right\rangle$, and relativistic energy factor $\langle\gamma\rangle$ on axial distance $z$. In the first section $(z=0 \sim 12 \mathrm{~cm})$, the transverse velocity and radial coordinate rapidly increase after the beam gets trapped. The axial magnetic field also increases as the beam energy increases. In the second section $(z=12 \sim 52 \mathrm{~cm})$, the magnetic field changes very little although the beam energy goes up fast. That is because increase of refractive index balances increase of beam energy as the waveguide radius gets larger, which can be seen from Eq. 1. In the third section $(z=52 \sim 112 \mathrm{~cm})$, the average radial coordinate $\langle r\rangle$ does not go up but oscillates while the beam energy keeps increasing, with a maximum voltage of $1.085 \mathrm{MV}$ at the end. The oscillation results from the deviation of guiding centers of electrons which have a nearly constant gyration radius (see Fig. 1). In the drift section $(z=112 \sim 172 \mathrm{~cm})$, the axial velocity increases and the transverse velocity decreases, holding constant the adiabatic invariant $\beta_{\perp}^{\perp} / B_{o}$ when $B_{o}>600 \mathrm{G}$. When $B_{o}<600 \mathrm{G}(z>152 \mathrm{~cm})$, the adiabatic condition begins to be violated. Finally, the "self-scanning" electron beam leaves the drift region with $\left\langle\beta_{\perp}\right\rangle=0.2055$ and $<\beta_{z}>=0.9244$, with a scanning cone angle of about 11.5 degrees, as shown in Fig. 2.
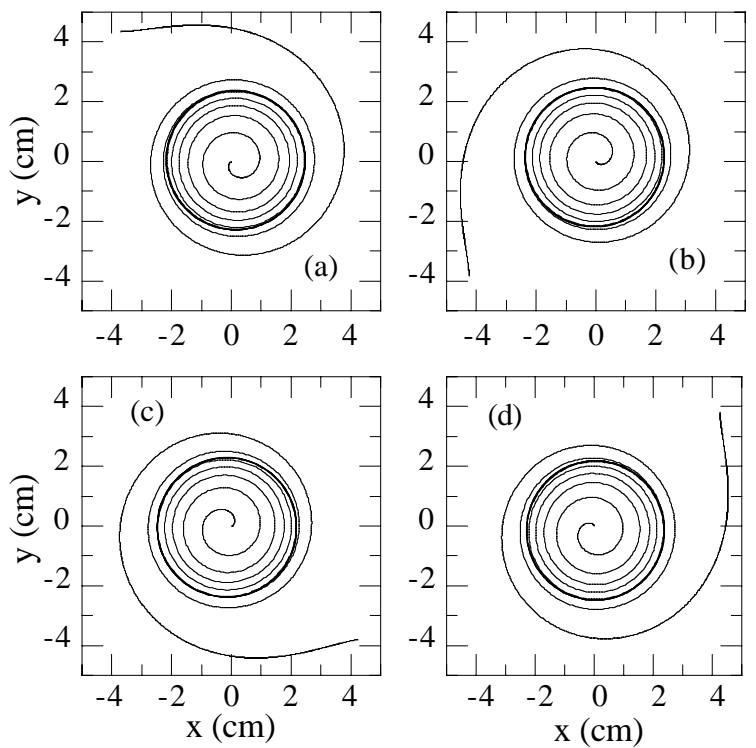

Figure 4: Orbits on the $x-y$ plane for four sample particles with different initial phases.

The feature of "self-scanning" of the beam emerging from CARA is shown in Fig. 4, with orbits in the $x-y$ plane for four sample particles with relative initial timephases of 0 (a), $\pi / 2(\mathrm{~b}), \quad \pi(\mathrm{c})$, and $3 \pi / 2$ (d) respectively. Several orbit turns overlap after the electrons enter CARA, in making a few revolutions. On the overlapped orbits, the gyration radius is almost the same, as mentioned previously. Then the electrons pass into the drift region and their radial coordinates get larger and larger as the magnetic field gets weaker and weaker.
Fig. 5 shows the dependence of electron beam power $P_{b}$ and $\mathrm{rf}$ drive power $P_{r f}$ on axial distance $z$. It is seen that $21-\mathrm{MW}$ drive power at $2.856 \mathrm{GHz}$ is decreased down to $90 \mathrm{~kW}$ at the end of CARA, while the beam power is increased from $6.25 \mathrm{MW}$ to $27.125 \mathrm{MW}$, with an rf conversion efficiency of over $99 \%$. The wall loss is seen to be minuscule, just $35 \mathrm{~kW}$, since CARA operates at a relatively low drive frequency.

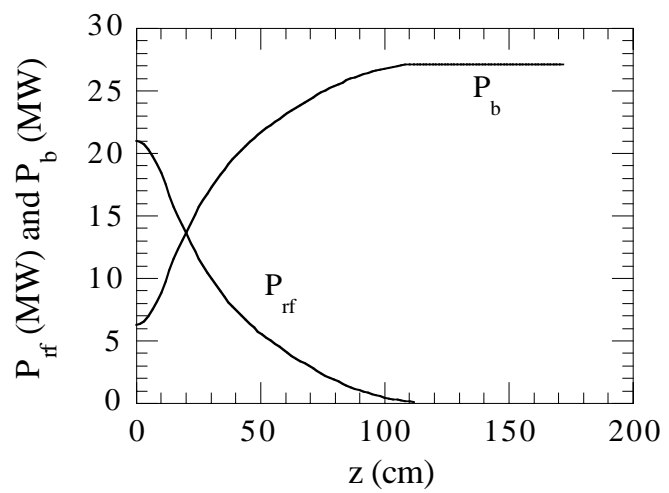

Figure 5: Dependence of electron beam power $P_{b}$ and if drive power $P_{r f}$ on axial distance $z$.

\section{CONCLUSIONS}

Simulations have shown that a $250-\mathrm{kV}, 6.25-\mathrm{MW}$ electron beam can be accelerated up to a $1.08 \mathrm{MV}, 27$ MW "self-scanning" beam by the CARA, with an rf conversion efficiency of over 99\%. Stanford Linear Collider (SLC) $65 \mathrm{MW}, 2.856 \mathrm{GHz}$ klystrons have 3.5 $\mu s$ pulse width and $180 \mathrm{sec}^{-1}$ pulse repetition rates [8]. From this simulation, it can be expected that two SLC klystrons driving a future CARA with a 250-kV, 40-MW injected beam should allow generation of a beam suitable for EBDS with average power approaching $100 \mathrm{~kW}$.

\section{REFERENCES}

[1] See, for example, Radiation Physics and Chemistry 24, 1-201 (1984).

[2] Radiation for a Clean Environment, IAEA, Vienna (1975).

[3] R. J. Woods and A. K. Pikaev, Applied Radiation Chemistry: Radiation Processing (Wiley, New York, 1994); J. W. T. Spinks and R. J. Woods, An Introduction to Radiation Chemistry, 3rd ed. (wiley, New York, 1990).

[4] B. Hafizi, P. Sprangle, and J. L. Hirshfield, Phys. Rev. E 50, 3077 (1994).

[5] C. Wang and J. L. Hirshfield, Phys. Rev. E 51, 2456 (1995).

[6] M. A. LaPointe, R. B. Yoder, C. Wang, A. K. Ganguly, and J. L. Hirshfield, Phys. Rev. Lett. 76, 2718 (1996).

[7] C. Wang, J. L. Hirshfield, and A. K. Ganguly, in this proceedings.

[8] G. Caryotakis, IEEE Trans. Plasma Sci. PS-22, 683 (1994). 